\title{
The urologist and medical imaging
}

\author{
Ahmad Shabsigh and Peter T Scardino
}

Imaging ranks along with anesthesia and antibiotics as one of the major medical innovations of the past two centuries. Five years before Roentgen discovered X-rays, urology became a separate specialty, and Felix Guyon was appointed the first Professor of Urology. Since then, urologists have been at the forefront of genitourinary imaging. Urologists initially used plain abdominal films for the diagnosis of stones, and first injected contrast agents cystoscopically, for retrograde pyelograms, in the early 20th century. In 1929, urologist Moses Swick reported the first excretory urogram, which went on to become a routine office procedure for urologists for decades. In 1955, urologist Willard Goodwin performed the first percutaneous nephrostomy under image guidance. Urologists pioneered the application of transrectal ultrasonography of the prostate, which revolutionized the technique for needle biopsy - now one of the most widely performed image-guided diagnostic procedures in the world. Urologists also perform cryotherapy and high-intensity focused ultrasound ablation of prostate cancer under ultrasound guidance, and routinely use fluoroscopic imaging during extracorporeal shock-wave lithotripsy and percutaneous nephrolithotripsy, developments which have made open surgery for stones obsolete. Office urologists use ultrasound to scan the bladder for postvoid residual urine, and, in some countries, use abdominal ultrasound to screen for renal tumors in adults and for congenital anomalies in children. Medical imaging has been an essential tool of urology since its inception.

The new frontier for image-guided intervention in urology is percutaneous ablation of small renal masses. Interventional radiologists and urologists have independently, and sometimes jointly, reported their experience with this procedure. A debate is now developing about who should perform these procedures, and whether candidates for such procedures even need to see a urologist. This debate is likely to intensify as image-guided procedures become more sophisticated. MRI-guided needle biopsy ....a procedure

should be

carried out by

the physicians

who best

perform it

....a multi-

disciplinary

team might be

preferable.

A Shabsigh is a Fellow in Urologic Oncology at the Memorial Sloan-Kettering Cancer Center, NY, USA. PT Scardino is Editor-in-Chief of Nature Clinical Practice Urology.

\section{Competing interests}

The authors declared they have no competing interests.

www.nature.com/clinicalpractice doi:10.1038/ncpuro0461 of the prostate and brachytherapy have been reported, and MRI-guided high-intensity focused ultrasound will probably become more widely used in the next decade.

In fact, radiologists and urologists both bring value to the management of patients who are candidates for image-guided intervention. Radiologists bring training in the performance and interpretation of diagnostic imaging, knowledge of the capability of imaging technology, and skill at placing needles in a variety of abdominal lesions. Urologists bring knowledge of the disease and of the anatomy and pathology of the organ, skill with a variety of interventions, and experience with the alternative (medical, surgical, and minimally invasive) treatment options, which can help patients select the most appropriate timing and type of treatment. In addition, urologists can provide continuity of care, manage complications, and document outcomes.

Some policy makers and professional organizations argue that imaging studies should only be performed by certified radiologists. In medicine, turf battles and legislative mandates rarely lead to the best-quality care. By their very nature, improvements in medical care come about serendipitously, and might happen more rapidly when there is dynamic tension, and some competition, between specialties. In the final analysis, a procedure should be carried out by the physicians who best perform it, and a multidisciplinary team might be preferable.

Close collaboration between interventional radiologists and urologists will certainly help bring this new technology to our patients safely and quickly. How this will evolve in any given medical center will depend on the personality and vision of the leaders in these specialties. Those institutions that become paralyzed by internecine rivalries might find themselves relegated to the backwater of the development and application of these exciting advances in patient care. 